\title{
Diagnosis and management of carotid atherosclerosis
}

We have been alerted to an error in this Clinical Review by Ankur Thapar and colleagues (BMJ 2013;346:f1485, doi:10. 1136/bmj.f1485). In the legend under figure 2, STA should have been spelt out as "superior thyroid artery" [not "superficial temporal artery" as published].

\section{Cite this as: BMJ 2013;346:f2420}

๑ BMJ Publishing Group Ltd 2013 